\title{
Incidence of Maternal "Near-Miss" Events in a Tertiary Care Hospital of Central Gujarat, India
}

\author{
Niyati T. Parmar ${ }^{1} \cdot$ Ajay G. Parmar ${ }^{1} \cdot$ Vihang S. Mazumdar $^{1}$
}

Received: 29 December 2015/Accepted: 12 April 2016/Published online: 13 June 2016

(C) Federation of Obstetric \& Gynecological Societies of India 2016

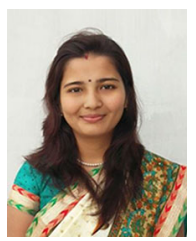

\begin{abstract}
About the Author
Niyati T. Parmar has graduated (MBBS) from Baroda Medical College, The Maharaja Sayajirao University, Vadodara, Gujarat, and completed her post-graduation (MD PSM) from the same college. At present, she is a Tutor in the Department of Preventive and Social Medicine at Medical College Baroda. Since last three years, she has involved in rural health activities under Rural Health Training Centre (RHTC) of Baroda Medical situated in Sokhada village near Vadodara city, with special focus on Maternal and Child Health activities. Study was carried out as a part of her thesis focusing on issues related to "Maternal Near-Miss" events.
\end{abstract}

\begin{abstract}
Background Constant decline in maternal mortality ratio has given rise to the need of a new indicator. This search has motivated investigation of severe maternal morbidity"maternal near-miss" (MNM) event. World Health Organization (WHO) defines MNM as "a woman who, being close to death, survives a complication that occurred during
\end{abstract}

Dr. Niyati T. Parmar, Tutor in Department of PSM at Medical College Baroda; Dr. Ajay G. Parmar, Tutor in Department of PSM at Medical College Baroda; Dr. Vihang S. Mazumdar, Professor and Head of Department of PSM in Department of PSM at Medical College Baroda.

Niyati T. Parmar dr.niyati251@gmail.com

1 Department of PSM, Medical College Baroda, Raopura, Vadodara, Gujarat 390001, India pregnancy, delivery or up to 42 days after the end of her pregnancy".

Methodology A hospital-based cross-sectional study was carried out at Sir Sayajirao General Hospital (SSGH), a tertiary care referral hospital in Vadodara, Central Gujarat, from May to September 2012. MNM events were identified according to either WHO or Mantel et al. criteria or both. Results During study period, 1929 live births, 18 maternal deaths and 46 "near-miss" cases were recorded. Among these 46 near-miss cases, 57 near-miss events were identified. Calculated MNM ratio was 23.85/1000 live births, MNM rate was 20.6/1000 obstetric admissions, the ratio of maternal death to MNM event was 1:2.6, and overall Mortality index was $28.1 \%$. Among near-miss cases, percentage of preterm delivery was $42 \%$ and stillbirth rate was $35 \%$ (16/46). Out of 46 MNM, pregnancies were continued in 3 while 43 pregnancies were terminated. (25 live births, 16 stillbirths, 2 abortions). 
Conclusion Identification of preventable factors and special preventive actions should be taken for management of complications in such near-miss cases.

Keywords Maternal near-miss (MNM) .

Maternal mortality · Central Gujarat · Government hospital

\section{Background}

Pregnancy-related mortality and morbidity continue to have a huge impact on the lives of Indian women and their newborn. Each year in India, roughly 28 million women experience pregnancy and 26 million have a live birth. Of these, an estimated 67,000 maternal and one million newborn deaths occur every year [1].

The maternal mortality ratio (MMR) is the most sensitive indicator for social inequalities. No other health indicator varies so dramatically between developed and developing countries. Reducing maternal mortality to 109 per 100,000 live births is the Millennium Development Goal 5 [2].

Maternal mortality is used as a sentinel event to assess the quality of a healthcare system. Maternal death review (MDR) is useful in improving the quality of obstetric care and reducing maternal mortality and morbidity [3]. Studies of negative outcomes have been highly successful in preventing causes responsible for maternal deaths. But this strategy of prevention faces problems when the numbers of negative outcomes drop to very low level [4]. MMR in India has shown an appreciable decline up to $178 / 100,000$ live births as per Sample Registration Survey 2010-2012 report [5]. As per latest MMR Bulletin 2011-2013, MMR of India showed decline up to $167 / 100,000$ live births [6].

Constant decline in the number of maternal deaths reflects a need for the search of a new indicator. This has motivated investigators to study hospital obstetrical morbidity data [7]. Various studies have revealed that maternal mortality represents the tip of an iceberg [8]. For each death, many other women survive serious complications during pregnancy, delivery and the puerperium. Investigating this group of women will increase the understanding of failures in obstetrical care within the healthcare systems of developing countries.

Survey of severe morbidity (near-miss) is one of the different approaches useful for investigation of maternal deaths [1].

The term "near-miss" describes a serious adverse event that only failed to occur by luck or by chance or by adequate management. This concept was defined by the World Health Organization (WHO) as "a woman who, being close to death, survives a complication that occurred during pregnancy, delivery or up to 42 days after the end of her pregnancy" $[8,9]$. Maternal near-miss (MNM) cases occur more often than maternal deaths and may give more information because the woman herself can be a source of data [10]. MNM cases have similar pathways as maternal deaths, with the advantages of offering a larger number of cases for analysis, greater acceptability of individuals and institutions since death did not occur [11].

There is limited information regarding incidence of and literature related to MNM as not many studies have been carried out in India. With this purpose, the present study was carried out at a tertiary care hospital of Central Gujarat. An attempt has been made to compare the WHO [12] and Mantel et al. [13] classifications (for identification and evaluation of MNM incidence) in terms of their applicability in public health programmes. The identification of near-miss events and their reasons can serve to identify the actions that can be taken to avert preventable maternal deaths.

Hitherto, three criteria; Mantel et al. [13], Waterstone et al. [12] and WHO [12], have been proposed for classifying maternal "near-miss" events. The present study tries to describe the incidence of near-miss in Sir Sayajirao General Hospital (SSGH)—regional referral hospital of central Gujarat.

\section{Aim}

To study maternal near-miss cases in a tertiary care hospital of Central Gujarat.

\section{Objectives}

1. To identify incidence of maternal "near-miss" event at a tertiary care hospital, Vadodara.

2. To compare the incidence of maternal near-miss as classified by WHO and Mantel et al. criteria.

3. To study maternal-foetal outcomes among the patients admitted at a tertiary care hospital, Vadodara.

\section{Methodology}

A hospital-based cross-sectional study was carried out at Department of Obstetrics and Gynaecology at Sir Sayajirao General Hospital (SSGH), Vadodara, the only government tertiary care referral hospital in Central Gujarat attached to medical college where the patients are referred from even remote tribal areas. Data collection was done from May to September 2012 for a period of six months. Selection of study participants was done from the Obstetrics ward 
irrespective of the place of delivery. Data collection was done through in-depth interviews of MNM mothers, 2 days after their admission, to ensure survival. The participants were followed up till their discharge. All the interviews were taken in local language in a semi-structured questionnaire prepared by the investigator and were taken by the same investigator to avoid inter-observer bias. MNM was defined using WHO [12] and Mantel et al. [13] criteria. Since both differ in their components, it was decided to use both. Privacy and anonymity of the participants was maintained throughout the study. Monthly and yearly statistics of all obstetrics events like total number of admission, total number of confinements, maternal deaths, number of complicated pregnancy, term or preterm delivery were obtained from Department of Obstetrics and Gynaecology and Medical Record and Statistical Department (MRSD) of SSG Hospital. Data entry and analysis were done with the help of Epi-Info software (version 6.04d) [14].

\section{Ethical Issues}

Before starting enrolment of the participants, necessary clearances and permissions were obtained from concerned authorities including Scientific and Ethical Research Committee (SERC) for Human research. At the time of data collection, the purpose of the study was clearly explained to the study subjects, and they were also assured of the confidentiality of the information. The participants were enrolled in the study only after taking written informed consent.

\section{Results}

Out of the 2238 obstetrics patients admitted in Obstetrics and Gynaecology ward during the study period, 2104 patients delivered at the hospital, and the remaining were admitted as ante-partum or post-partum cases. There were no refusals during entire study period. A total of 1929 live births, 18 maternal deaths and 46 "near-miss" cases (according to either WHO [12] or Mantel et al. [13] or both) were recorded. Fifty-seven near-miss events were identified among 46 near-miss cases, which mean that 11 women had more than one event, yielding a mean of 1.2 near-miss events per case.

Table 1 shows various indicators proposed by WHO to describe maternal events. Depending on the criteria used, incidence of severe maternal morbidity/near-miss events was 19.7 cases per 1000 live births as per Mantel et al. criteria and 20.7 cases per 1000 live births as per WHO criteria. This gives an overall MNM ratio of 23.85/1000 live births. MNM rate was calculated as 20.6/1000 obstetric admissions, and maternal mortality ratio was 933/100,000 live births for SSG Hospital. The ratio of maternal death to maternal near-miss event was 1:2.6, and overall Mortality index was $28.1 \%$. There were 50 women identified with suspected severe maternal morbidity, of which, 46 were identified as MNM according to either criterion. There were four cases that did not fit into either criterion. However, their clinical condition was so severe that they were clinically treated as near-miss [7]. Out of 46 near-miss mothers, 32 mothers fitted into both WHO and Mantel criteria, while eight patients fulfilled WHO criteria and six patients fulfilled Mantel et al. criteria only (Table 2). Cohen's kappa test was applied to test the agreement between these two criteria. Kappa value measures the proportion of non-random agreement between two tests, two methods or two observers. On applying this test, values obtained are as below:

Kappa $=0.817 \quad \mathrm{SE}=0.0479 \quad \mathrm{CI}=0.723-0.911$

Value of kappa greater than 0.75 is considered excellent. This indicates that there is strong or excellent agreement in identifying cases by both the criteria.

Tables 3 and 4 shows distribution of cases by Mantel et al. and WHO criteria. Most of the patients fulfilled both the criteria, i.e. Mantel et al. [13] and WHO [12]. According to Mantel et al. criteria [13], maximum numbers

Table 1 Indicators proposed by WHO to describe maternal events

\begin{tabular}{llll}
\hline Indicators & Overall (identified by both criteria) & WHO & Mantel et al. \\
\hline Absolute number of near-miss cases & 46 & 40 & 38 \\
Maternal near-miss rate/1000 obstetric admission & 20.55 & 17.87 & 16.98 \\
Maternal near-miss ratio/1000 live births & 23.85 & 20.74 & 19.7 \\
Ratio of maternal near-miss event to maternal death & 2.6 & 2.2 & 3.1 \\
Mortality index $(\%)^{\mathrm{a}}$ & 28.1 & -03 & 32.14 \\
Maternal mortality rate/1000 obstetric admission & 8.04 & - & - \\
Maternal mortality ratio/1,00,000 live births & 933 & - & - \\
\hline
\end{tabular}

${ }^{a}$ Mortality index (MI): number of maternal death/number of maternal death + number of near-misses $\times 100(\%)$ 
Table 2 Comparison of maternal near-miss events by WHO and Mantel et al. criteria

\begin{tabular}{lccc}
\hline Mantel et al. & \multicolumn{2}{l}{ WHO criteria } & Total \\
\cline { 2 - 3 } & Satisfied & Not satisfied & \\
\hline Satisfied & 32 & 6 & 38 \\
Not satisfied & 8 & 4 & 12 \\
Total & 40 & 10 & 50 \\
\hline
\end{tabular}

Kappa $=0.817, \mathrm{SE}=0.0479, \mathrm{CI}=0.723-0.911$

Table 3 Distribution of cases by Mantel et al. criteria [13] $(\mathrm{n}=38)$

\begin{tabular}{ll}
\hline Criteria & $\begin{array}{l}\text { No. of } \\
\text { patients }^{\mathrm{a}}\end{array}$ \\
\hline $\begin{array}{l}\text { Admission to the intensive care unit (ICU) for sepsis or } \\
\text { other causes }\end{array}$ & 14 \\
$\begin{array}{l}\text { Hypovolemia (requiring } 5 \text { or more units of whole blood or } \\
\text { packed cells for resuscitation) }\end{array}$ & 13 \\
$\begin{array}{l}\text { Emergency hysterectomy } \\
\text { Acute thrombocytopenia requiring platelet transfusion }\end{array}$ & 9 \\
$\begin{array}{l}\mathrm{O}_{2} \text { saturation below } 90 \% \text { for more than } 60 \text { min } \\
\text { Ventilation for more than } 60 \text { min, except for general } \\
\text { anaesthesia }\end{array}$ & 4 \\
Urine output less than $400 \mathrm{ml} / 24$ h, refractory to hydration, \\
$\quad$ furosemide or dopamine & 1 \\
$\begin{array}{l}\text { Acute deterioration of BUN and creatinine (>15 mol and } \\
\quad>400 \text { mol) }\end{array}$ & 1 \\
$\begin{array}{l}\text { Jaundice with pre-eclampsia } \\
\text { Patient mayt }\end{array}$ & 1
\end{tabular}

${ }^{a}$ Patient may fit into multiple criteria

of patients required ICU admission, the most common indication being ante-partum eclampsia, and others being intra-partum eclampsia, ante-partum haemorrhage, PIH (pregnancy-induced hypertension) with heart disease, RHD (rheumatic heart disease), chorioamnionitis and hepatic coma. The WHO classification is remarkable for identifying the most serious cases with higher risk of death. At the same time, WHO classification showed a very high threshold for the detection of cases of MNM, missing a significant proportion of women with conditions such as pre-eclampsia and eclampsia.

Majority of the patients missed by Mantel et al. $(N=8)$ fit into WHO criteria of bilirubin $>100 \mathrm{mmol} / \mathrm{L}$ or $>6.0 \mathrm{mg} / \mathrm{dL}$. The average total bilirubin was $10.8 \mathrm{mg} / \mathrm{dl}$. All these patients had yellowish discoloration of urine and sclera for 8-10 days. Two patients suffering from jaundice had hepatitis E. Another important set of patients missed by Mantel et al. criteria were patients suffering from coagulation disorder. There were four "near-miss" patients who had coagulation disorder (DIC-disseminated intravascular coagulation).
Table 4 Distribution of cases according to WHO criteria [12] $(n=40)$

\begin{tabular}{lc}
\hline & $\begin{array}{l}\text { No. of } \\
\text { patients }^{\mathrm{a}}\end{array}$ \\
\hline Clinical criteria & 4 \\
Coagulation disorders & 2 \\
Loss of consciousness for $\geq 12 \mathrm{~h}$ & 1 \\
Jaundice with pre-eclampsia & \\
Laboratory criteria & 8 \\
Bilirubin $>100$ mmol/L or $>6.0 \mathrm{mg} / \mathrm{dL}$ & 8 \\
Acute thrombocytopenia $(<50000$ platelets) & 4 \\
Oxygen saturation $<90 \%$ for $\geq 60 \mathrm{~min}$ & 1 \\
Creatinine $\geq 300$ mmol/L or $\geq 3.5 \mathrm{mg} / \mathrm{dL}$ & 11 \\
Management criteria & 10 \\
Transfusion of $\geq 5$ units of PRBCs & 1 \\
Hysterectomy for post-partum haemorrhage or infection & 3 \\
Dialysis for acute renal failure & \\
Intubation and ventilation for $\geq 60$ min not related to \\
anaesthesia
\end{tabular}

\section{Maternal-Foetal Outcome}

Out of $22(\sim 50 \%)$ MNM patients admitted during antenatal period, 14 patients presented before 37 weeks of pregnancy. Of them, pregnancy was continued in only two patients, while 12 out of 14 patients $(86 \%)$ ended up with premature delivery. Out of 15 patients, being admitted during intra-partum period, labour started before 37 weeks of pregnancy in four patients and ended up with preterm delivery. Nine patients delivered outside SSGH and were referred here for management of post-partum complications.

Table 5 shows that among near-misses cases, percentage of preterm delivery was $42 \%$ while it was $27 \%$ in total obstetric admissions. Out of 18 patients in whom preterm delivery occurred, 14 patients came with complication during antenatal period and pregnancy needed to be terminated. While four patients came with premature rupture of membrane (PROM) and ended up with preterm delivery. Proportion of term delivery was 58 and $73 \%$ among nearmisses and in total obstetric admission, respectively. Delivery was conducted in 43 out of 46 MNM cases (25 live births, 16 stillbirths, 2 abortions), and pregnancy was continued in three mothers after appropriate treatment. Of these 43 MNM, 17 delivered by normal vaginal delivery, 17 by caesarean section, one by assisted vaginal and eight by emergency laparotomy. The absolute percentage of normal delivery and caesarean section was similar (50-50 \%) among MNM, while it was 66.7 and $33.3 \%$ among total confinement, respectively (Table 5). 
Table 5 Comparison of events between near-misses and total confinement during same study period (from data available)

\begin{tabular}{|c|c|c|c|c|c|}
\hline Event & Among near-misses & $\%$ & Among total confinement & $\%$ & $P$ value \\
\hline Preterm delivery & $18(n=43)$ & 41.86 & $453(n=1657)$ & 27.34 & 0.0538 \\
\hline Stillbirths & $16(n=41)$ & 39.02 & $200(n=2129)$ & 9.39 & $<0.0001$ \\
\hline Live births & $25(n=41)$ & 60.98 & $1929(n=2129)$ & 90.61 & \\
\hline Patients delivered by caesarean section & $17(n=34)$ & 50 & $635(n=1908)$ & 33.28 & 0.0625 \\
\hline Patients delivered by normal delivery & $17(n=34)$ & 50 & $1273(n=1908)$ & 66.72 & \\
\hline
\end{tabular}

\section{Discussion}

The study describes results of a hospital-based investigation of maternal near-miss events. Out of three existing criteria, the WHO [12] and Mantel et al. [13] classifications were used in the study to identify and evaluate the incidence of maternal near-misses. An attempt has been made to compare both in terms of their applicability in public health programmes. Waterstone $\mathrm{M}$ et al. criteria were not used in our study as in some instances identifying "nearmiss" using these criteria overestimates near-miss cases. During this study, depending on the criteria used, overall maternal near-miss ratio was found to be $23.85 / 1000$ live births, which is within the range of ratios $(12.3-82.3$ per 1000 deliveries) reported in various studies using similar criteria for near-miss definition $[15,16]$

Observed ratio of maternal near-miss event to maternal death was 2.6 in our study, while it was 8.6 in a study conducted by Fátima A L et al. in Brazil [8].

These ratios, however, cannot be applied for programme or other centres because patients who are likely to be nearmiss or potential maternal mortality cases are preferentially referred to tertiary care hospitals. This can be attributed to "referral biases".

As shown in results, value of kappa obtained was 0.817 , which indicates that there is strong or excellent agreement in identifying cases by WHO and Mantel et al. criteria. In this study, percentage of preterm delivery was $42 \%$ among near-misses as compared to $27 \%$ in total obstetric admission which was statistically significant $(p=0.05)$. Proportions of live and stillbirths among total near-misses were $54 \%(25 / 46)$ and $35 \%$ (16/46), respectively. Excluding all those MNM who aborted or in whom pregnancy was continued after giving proper treatment, counted percentage of live births were $61 \%$ (25/41), and stillbirths were $39.02 \%$ (16/41) [Table 5]. Analytic results indicate that difference observed between outcome during pregnancy (live birth and stillbirth) among near-misses and among total obstetric admission is highly significant $(p<0.0001)$. Complications arising in near-miss cases like $\mathrm{APH}, \mathrm{PIH}$ may be the reason behind higher stillbirths. Absolute percentage of normal delivery was also lower (50\%) among near-misses as compared to $66.7 \%$ in general. Similarly, percentage of caesarean section was much more $(50 \%)$ as compared to rate of caesarean section $33.3 \%$ in general. In a study by Fátima et al., $85.8 \%$ of the deliveries were performed by caesarean section (121/ 141 deliveries) [8].

\section{Conclusion and Recommendation}

The near-miss rate in hospital setting was 20.6 per 1000 obstetric admission and near-miss ratio was 23.85 per 1000 live births. Depending on the criteria used, incidence of severe maternal morbidity/near-miss events in our study was 19.7 cases per 1000 live births as per Mantel criteria and 20.7 cases per 1000 live births as per WHO criteria. Though both the WHO or Mantel criteria are different, there is no statistically significant difference in identifying MNM cases by either criterion. Rate of preterm delivery and percentage of caesarean section was higher among nearmisses as compared to total confinement, which was 42 and $50 \%$, respectively. MNM assessment is a useful tool to identify factors associated with maternal complications as well as those which associated with survival.

Study of factors leading to near-miss events may be factors related to maternal mortality also. Taking this into consideration, identification of preventable factors and special preventive actions should be included for management of complications in such cases. A study of MNM and its associated factors helps us identify what things were done correctly before referral which could have impacted survival and vice versa, and thereby help us in advocacy with referral facility to develop standard operating protocol (SOP) appropriate to presentation before referral. Now tailor-made new "maternal near-miss guideline" was made by Government of India [17]. This study highlights the need for proper implementation of it at peripheral as well as central levels. There is also a need for validation of these "maternal near-miss" criteria at peripheral levels. This may enable the peripheral healthcare system in early identification of such factors and help prevent maternal deaths.

Acknowledgments We are thankful to Dr. Chandresh Pandya, Associate Professor, Community Medicine Department and Dr. R K Baxi, Professor, Community Medicine Department, whose incessant 
efforts in guiding this study have been of pivotal importance. We are indebted to Dr Kalpita Shringarpure for their valuable support from inception to execution of this study and its final shape. We are thankful to the Head of Department, Obstetrics and Gynaecology, all resident doctors of Obstetrics and Gynaecology wards and Medical Superintendent of the hospital for their continuous support throughout the process of data collection and for allowing study and to use data for the study. We are highly obliged to all the study participants, who willingly and enthusiastically participated in the study; without their support, the study would not have been possible.

Funding This study was not funded by any kind of funding agency.

\section{Compliance with Ethical Standards}

Conflict of interest Dr. Niyati Parmar declares that she has no conflict of interest. Dr. Ajay Parmar declares that he has no conflict of interest. Dr. Vihang Mazumdar declares that he has no conflict of interest.

Ethical Approval Before starting enrolment of the participants, necessary clearances and permissions were obtained from concerned authorities including Scientific and Ethical Research Committee (SERC) for Human research. Scanned copy of which has been attached here with in additional document.

Informed Consent At the time of data collection, the purpose of the study was clearly explained to the study subjects and they were also assured of the confidentiality of the information. The study subjects were enrolled in the study only after taking written informed consent.

\section{References}

1. http://mohfw.nic.in/NRHM/Maternal_Health.htm.

2. The Millennium Development Goals Eight Goals for 2015. http://www.undp.org/content/india/en/home/mdgoverview/.

3. Maternal Death Review [database on the Internet]. Department of Health and Family Welfare, Government of Punjab. 2010. http://www.pbnrhm.org/docs/mdr.pdf.

4. Wikipediatfe.Maternalnearmiss. http://en.wikipedia.org/wiki/ Maternal_near_miss.

5. A presentation on maternal mortality levels (2010-2012) [database on the Internet]. 2013. www.censusindia.gov.in/.../MMR 2010-12-Report_Pres_19.12.2013.ppt.
6. Maternal Mortality Ratio Bulletin 2011-2013 [database on the Internet]. Government of India, Ministry of Home Affairs. http://www.censusindia.gov.in/vital_statistics/mmr_bulletin_201113.pdf.

7. Parmar N, Parmar A, Mazumdar VS, et al. What can we miss in identifying "maternal near miss" event? IJCRR. 2014;6(13): $45-50$.

8. Lotufo FA, Parpinelli MA, Haddad SM, et al. Applying the new concept of maternal near-miss in an intensive care unit. Clinics (Sao Paulo). 2012;67(3):225-30.

9. Say L, Souza J, Pattinson R, et al. WHO working group on maternal mortality and morbidity classifications. maternal near miss-towards a standard tool for monitoring quality of maternal health care. Best Pract Res Clin Obstet Gynaecol. 2009;23(3): 287-96.

10. Souza J, Cecatti J, Parpinelli M, et al. Appropriate criteria for identification of near-miss maternal morbidity in tertiary care facilities: a cross sectional study. BMC Pregnancy and Childbirth. 2007;7(1):20.

11. Amaral E, Souza J, Surita F, et al. A population-based surveillance study on severe acute maternal morbidity (near-miss) and adverse perinatal outcomes in Campinas, Brazil: the Vigimoma Project. BMC Pregnancy and Childbirth. 2011;11(1):9.

12. Morse ML, Fonseca SC, Gottgtroy CL, et al. Severe maternal morbidity and near misses in a regional reference hospital. Rev Bras Epidemiol. 2011;14(2):310-22.

13. Mantel GD, Buchmann E, Rees H, et al. Severe acute maternal morbidity: a pilot study of a definition for a near-miss. BJOG: Int J Obstet Gynaecol. 1998;105(9):985-90.

14. Dean AG, Arner TG, Sunki GG, et al. Epi_Info ${ }^{\text {TM }}$, a database and statistical programme for public health professionals on IBMcompatible microcomputers. Centers for Disease Control and Prevention. Atlanta, Georgia, USA. 2011.

15. Ali A, Khojali A, Okud A, et al. Maternal near-miss in a rural hospital in Sudan. BMC Pregnancy and Childbirth. 2011;11(1): 48.

16. Say L, Pattinson RC, Gulmezoglu AM, et al. WHO systematic review of maternal morbidity and mortality: the prevalence of severe acute maternal morbidity (near miss). Reprod Health. 2004;1(1):3.

17. Maternal Near Miss Review Operational Guidelines. In: Maternal Health Division, Ministry of Health \& Family Welfare. Government of India. 2014. http://www.nrhmorissa.gov.in/ writereaddata/Upload/Documents/Maternal_Near_Miss_Operational_ Guidelines. 